\title{
Microwave-assisted extraction of lycopene in tomato peels: Effect of extraction conditions on all-trans and cis-isomer yields
}

LWT - Food Science and Technology (accepted manuscript)

DOI: https://doi.org/10.1016/j.lwt.2014.12.061

Kacie K.H.Y. Ho, M.G. Ferruzzi, A.M. Liceaga, M. Fernanda San Martín-González*

Department of Food Science, College of Agriculture, Purdue University, 745 Agriculture Mall Drive, West Lafayette, IN, 47907, USA

*Corresponding Author

Keywords: All-trans-lycopene, Cis-isomers, Microwave-assisted extraction, Response surface methodology

\section{Highlights}

- Maximum all-trans-lycopene concentration was attained with ethyl acetate at $400 \mathrm{~W}$, with $24 \mathrm{~kJ}$ equivalents ( 1 min treatment).

- All-trans-lycopene yields were significantly higher with microwave-assisted versus conventional extraction.

- Microwave-assisted extraction appeared to substantially disrupt tomato cell structure, possibly improving lycopene yield. 


\title{
LWT - Food Science and Technology
}

Volume 62, Issue 1, Part 1, 2015, Pages 160-168

\begin{abstract}
Lycopene is the primary carotenoid in tomato peels, a processing byproduct, and can be used as a natural color or bioactive ingredient. Unfortunately, extractions are inefficient as lycopene is extremely nonpolar and susceptible to degradation. As a rapid technique, microwave-assisted extraction (MAE) potentially offers efficient lycopene recovery. Thus, the objectives of this research were to: 1) optimize MAE of lycopene from tomato peels and 2) evaluate the effect of treatment on all-trans and isomer yields. Response surface methodology (RSM) was employed to optimize lycopene extraction with solvent ratio solid-liquid ratios, microwave power, and delivered energy equivalents as factors. High performance liquid chromatography with a diode array detector (HPLC-DAD) was used for isomer separation and quantification. Optimum MAE conditions were determined as: 0:10 solvent ratio at $400 \mathrm{~W}$ with a yield of $13.592 \mathrm{mg} / 100 \mathrm{~g}$ of extracted all-trans-lycopene. RSM suggested that ethyl acetate was a better MAE solvent for lycopene recovery as compared to hexane, which overall extracted less lycopene. HPLC-DAD indicated that MAE significantly improved all-trans and total lycopene yields, while conventional extraction demonstrated higher proportions of cis-isomer yields. Additionally, electron micrographs showed that significant structural disruption occurred in MAE-treated samples, possibly allowing for the improved lycopene extraction.
\end{abstract}




\section{Introduction}

The tomato industry is a multi-billion dollar market with the US being a top producer of tomatoes for processed foods (Thornsbury, 2012). In 2009, production exceeded 13 million tons (Economic Research Service, 2010), of which, 12\% (the peel portion) was considered waste despite having more lycopene than the pulp by weight (Al-Wandawi et al., 1985, George et al., 2004). Lycopene, $\mathrm{C}_{40} \mathrm{H}_{56}$, is the primary pigment responsible for the red hue in tomatoes, watermelon, and blood oranges (Rodriguez-Amaya, 2001). As an acyclic, highly conjugated isoprenoid, lycopene is the most potent singlet oxygen quencher of all carotenoids (Di Mascio, Kaiser, \& Sies, 1989). Consumption of lycopene from tomatoes has been associated with protection against oxidative DNA damage and anticancer properties (Agarwal \& Rao, 2000), thus making it a compound of interest amongst medical and nutrition researchers.

Aside from potential health benefits, lycopene offers an alternative to synthetic food colorants. From a processing standpoint, extraction can be difficult as food grade solvent choices are limited. However, isolating lycopene from tomato peels can reduce the overall cost by adding value to an otherwise discarded waste product. Lycopene is insoluble in water and poorly soluble in organic solvents, which limits its removal from raw plant material. However, extraction efficiency of carotenoids can be improved by using solvent combinations to facilitate partitioning. Previous research indicated that solvent systems containing hexane and ethyl acetate are the most efficient for carotenoid extraction from tomato seeds and peels (Strati \& Oreopoulou, 2011). Despite improvements, the extraction procedure itself is time consuming and poses the risk of degradation as samples are exposed to heat for extended periods of time. Due to this limitation, pure lycopene is often expensive (Ascenso et al., 2013). Improvements in extraction efficiency or reduction in extraction time may reduce the processing costs while producing a high value color.

In its natural form, lycopene is heat resistant and present in a thermodynamically stable, alltrans, crystal within the chromoplasts of plant cells (Harris \& Spurr, 1969). Conventional extraction often requires heat to facilitate the migration of solvent to extract pigment compounds. Although increased temperatures correspond with improved solubility and organelle membrane disruption, heat exposure should be limited when possible due to the thermolabile nature of carotenoids once they are in solvent (Rodriguez-Amaya, 2001). Although lycopene has been shown to be more stable in general against isomerization and degradation compared to $\beta$ carotene (Nguyen \& Schwartz, 1998) previous studies have demonstrated that heat treatments, longer than $1 \mathrm{~h}$, favored the trans-to-cis isomer conversion of lycopene while light irradiation induced cis-isomer degradation over time in tomato products (Chen et al., 2009, Shi et al., 2008).

Microwave-assisted extraction (MAE) may provide a solution for this since this technology induces rapid heating primarily within polar constituents due to dipole rotation and ionic drifting (Neas \& Collins, 1988). In theory, superheating of polar cellular components will improve migration of lycopene into the extraction solvent, while the short treatment times limit heat exposure of the nonpolar components. Previously, MAE has been used to enhance extraction of catechins, anthocyanins and curcuminoids (Baiano et al., 2014, Dandekar and Gaikar, 2002, Zou et al., 2012) among others has improved efficiency compared to conventional extraction. Although MAE of various phytochemicals has been investigated, limited research has been done 


\section{LWT - Food Science and Technology}

Volume 62, Issue 1, Part 1, 2015, Pages 160-168

on the effect of MAE on cis vs. trans isomer yield. Thus, the objectives of this study were to 1) determine the optimal MAE conditions for lycopene from tomato peels using response surface methodology and 2) evaluate the effect of treatment on cis- and trans-lycopene yields.

\section{Materials and methods}

\subsection{Reagents and standards}

All-trans-lycopene standard and all reagents were purchased from Sigma Chemical Co. (St. Louis, MO). Solvents were purchased from J.T. Baker (Phillipsburg, NJ). Tomato peels were generously donated by a Red Gold Co. (Elwood, IN). To prevent light-induced degradation of lycopene, all extractions were done in yellow light and extraction solvents contained butylated hydroxytoluene (BHT) to limit oxidation occurring during the centrifugation and handling of the extracts.

\subsection{Raw materials and sample preparation}

Tomato peels were obtained from a local processing facility as a byproduct of tomato paste. During the tomato processing, caustic lye was used to remove peels. Consequentially, received tomato peels were collected in bulk and neutralized with hydrochloric acid until a pH of 7 was obtained. Excess moisture was removed by squeezing peels with a cheesecloth prior to storage. All samples were flushed with nitrogen and stored at $-20{ }^{\circ} \mathrm{C}$ until further processing.

Since smaller particle sizes better facilitate extraction, the peels were further processed prior to microwave treatment. Frozen peels were ground using a spice grinder until a particle size of $<0.5 \mathrm{~cm}$ was achieved. The moisture content of the ground peels was analyzed with a MAX2000 Computrac Moisture Analyzer (Arizona Instruments, Chandler, AZ USA). Ideally, the moisture content of each sample should be quantified, however, due to the destructive nature of moisture analysis, the frozen supply of ground tomato peels were sampled from ten different locations within the sample stock. The mean value $(70.345 \pm 1.405)$ was later used to calculate the extraction yield of lycopene per weight of tomato peel on a dry weight basis. Although using the mean moisture content is not the best way to express the data, the variability between sampled portions was low $(<2 \%)$.

Peels were not dried as the water present increased polarity, which could aid in selective heating during microwave irradiation. Ground peels were stored in glass, screw top bottles, flushed with nitrogen, and stored at $-20^{\circ} \mathrm{C}$ until treated.

\subsection{Experimental design}

Response surface methodology (RSM) was used to determine the effect of extraction parameters on lycopene yield. Initially, RSM was conducted to assess four factors, solvent ratio $\left(X_{1}\right)$, solidliquid ratio $\left(X_{2}\right)$, microwave power $\left(X_{3}\right)$, and energy equivalents $\left(\mathrm{X}_{4}\right)$, which were varied by adjusting treatment time, with a Box-Behnken design comprising of 3 center points (Table 1). A secondary RSM was employed to investigate solvents containing a higher ethyl acetate (EA) percentage. For this only two factors, solvent ratio $\left(X_{1}\right)$ and microwave power $\left(X_{2}\right)$, were studied with a central composite design (CCD) with two center points (Table 1). A second-order 
polynomial equation (Eq. (1)) was used to express the response yield of all-trans-lycopene and cis-lycopene $\left(Y_{i}\right)$ as a function of the experimental factors $\left(X_{i}\right)$ for each RSM design:

$$
Y_{i}=b_{0}+\sum_{i=1}^{n} b_{n} x_{n}+\sum_{i=1}^{n} b_{m n} x_{n}^{2}+\sum_{i=1}^{n} b_{n m} x_{n} x_{m}
$$

where $b_{0}$ is a constant, $b_{n}, b_{m n}$, and $b_{n m}$ are the linear, quadratic, and interaction coefficients, respectively. The multiple regression models were analyzed separately for each $\mathrm{Y}_{\mathrm{i}}$, such that one response was a function of four (low EA) or two (high EA) independent variables. The model was predicted using regression analysis and analysis of variance (ANOVA).

Table 1. Response surface methodology parameters.

\begin{tabular}{|c|c|c|c|c|c|c|}
\hline \multirow{3}{*}{ Factor } & \multicolumn{3}{|c|}{ Low EA Experiments } & \multicolumn{3}{|c|}{ High EA Experiments } \\
\hline & \multicolumn{3}{|c|}{ Coded Value } & \multicolumn{3}{|c|}{ Coded Value } \\
\hline & -1 & $\mathbf{0}$ & 1 & -1 & $\mathbf{0}$ & 1 \\
\hline $\begin{array}{c}\text { Solvent ratio (mL } \\
\text { Hexane : mL Ethyl } \\
\text { acetate) }\end{array}$ & $1: 0$ & $1.5: 0.5$ & $1: 1$ & $2: 8$ & $1: 9$ & $0: 1$ \\
\hline $\begin{array}{l}\text { Solid-liquid ratio } \\
\qquad(\mathrm{g} / \mathrm{mL})\end{array}$ & $1: 20$ & $2: 20$ & $4: 20$ & \multicolumn{3}{|c|}{ N/A; Fixed at $1: 20 \mathrm{~g} / \mathrm{mL}$} \\
\hline Power (W) & 400 & 800 & 1600 & 400 & 800 & 1600 \\
\hline Energy $(\mathrm{kJ})$ & 24 & 36 & 48 & \multicolumn{3}{|c|}{ N/A; Fixed at $24 \mathrm{~kJ}$} \\
\hline
\end{tabular}

\subsection{Microwave-assisted extraction of lycopene}

Ground tomato peels were thawed to room temperature and weighed into teflon-lined extraction vessels at 1, 2, or $4 \mathrm{~g}$. Precisely $20 \mathrm{~mL}$ of corresponding solvent was added with a magnetic stir bar prior to capping. A Mars Xpress microwave extraction system (CEM Corp., Matthews, NC) was used at 400,800 , and $1600 \mathrm{~W}$ at varying times to achieve delivered energy equivalents of 24,36 , and $48 \mathrm{~kJ}$. Within the closed microwave system, 8 extraction vessels were arranged in a carousel following CEM Corp. protocol. Although 8 vessels were irradiated, only three vessels were sampled and analyzed as the triplicates per treatment.

Approximately $10 \mathrm{~mL}$ of saturated sodium chloride in water was added to the treated samples to facilitate partitioning and to break emulsions formed at the interface. This was then transferred to a $50 \mathrm{~mL}$ polypropylene tube and centrifuged in a 5804 centrifuge (Eppendorf, Hamburg, Germany) at 4,472 $\mathrm{g}$. The organic phase was collected and centrifugation was repeated with additional solvent two more times to ensure collection of all extracted lycopene. All organic phases were pooled, filtered through anhydrous sodium sulfate to remove residual water and adjusted to $50 \mathrm{~mL}$ prior to drying $2 \mathrm{~mL}$ aliquots under nitrogen and freezer storage, at $-20{ }^{\circ} \mathrm{C}$. Although direct injection would be more efficient, hexane was removed to prevent solvent effects during analysis. 


\subsection{Conventional extraction of lycopene}

Conditions used for the conventional extraction were selected to emulate the optimum conditions determined by response surface methodology. Conventional extraction was conducted with $1 \mathrm{~g}$ of ground tomato peels and $20 \mathrm{~mL}$ of a 1:1 (mL:mL) mixture of hexane (1 mg mL $\mathrm{mL}^{-1}$ BHT)-ethyl acetate in a $50 \mathrm{~mL}$ polycenrifuge tube. The tube was placed in a shaking water bath for $15 \mathrm{~s}$ at $45^{\circ} \mathrm{C}$, which falls within the temperature ranges observed for the optimal MAE treatment. Since conventional solvent extraction typically involves a longer heating time, another treatment was done following the same conditions, except the heat treatment was extended to $30 \mathrm{~min}$. The conventional methods used for high EA treatment (0:1 solvent ratio, 1:20 solid-liquid ratio, $400 \mathrm{~W}, 24 \mathrm{~kJ}$ equivalents for $1 \mathrm{~min}$ ) comparison followed the same protocol, except $20 \mathrm{~mL}$ of ethyl acetate ( $1 \mathrm{mg} \mathrm{mL}^{-1}$ BHT) was used as the solvent and heated for 1 min and $30 \mathrm{~min}$. Following heat treatment, the extraction process was the same as that done for MAE after microwave irradiation.

\subsection{Quantification with high performance liquid chromatography (HPLC-DAD)}

Carotenoid analysis was done using reversed phase HPLC-DAD based on the method used by Kean, Hamaker, and Ferruzzi (2008) using an Agilent 1200 Series HPLC, equipped with a diode array detector and a YMC Carotenoid S-3 C-30 column $(2.0 \times 150 \mathrm{~mm}, 3 \mu \mathrm{m}$ particle size $)$. A binary mobile phase of methanol with $2 \%$ aqueous ammonium acetate $(\mathrm{pH}=4.5)$ and ethyl acetate was used at a flow rate of $0.37 \mathrm{~mL} / \mathrm{min}$ with a gradient as follows: $0 \% \mathrm{~B}(0 \mathrm{~min}), 80 \% \mathrm{~B}$ (6 min), 100\% B (12 min), 0\% B (14 min). Precisely $10 \mu \mathrm{L}$ of sample was injected and lycopene was quantified at $470 \mathrm{~nm}$. Peak spectra were collected within the 200-600 nm range and analyzed with Chemstation software (Agilent Technologies, Santa Clara, CA).

Chromatograms of all-trans-lycopene standard yielded a peak at a retention time of $\sim 11 \mathrm{~min}$. Three cis-isomers of lycopene were separated and all-trans-lycopene and isomers were identified by comparing retention times with carotenoid profiles of a test salad containing known carotenoids (Goltz, Campbell, Chitchumroonchokchai, Failla, \& Ferruzzi, 2012) to rule out extraction of non-lycopene carotenoids. Since only trans-lycopene is readily available as a standard, cis-isomers were collectively quantified from the calibration curve of the all-translycopene. The 5-cis-isomer, which was observed as a pronounced shouldering peak off of translycopene, was quantified along with the other cis-isomers.

For calibration, a small amount of all-trans-lycopene standard was solubilized in petroleum ether to make a stock lycopene solution with an absorbance $\sim 0.8$. The absorbance of this solution and subsequent dilutions were read using a UV-Vis DU 800 spectrophotometer (Beckman and Coulter, Inc., Brea, CA) at $470 \mathrm{~nm}$. The stock solution was diluted to concentrations between 6.0 and $0.04 \mu \mathrm{M}$. The absorbance, done in triplicate, was then used to calculate the concentration of the stock and six dilutions with a molar extinction coefficient of $1.85 \times 10^{5} \mathrm{~L} \mathrm{~mol}^{-1} \mathrm{~cm}^{-1}$. Each lycopene dilution was dried under nitrogen and analyzed with HPLC-DAD to correlate peak area with lycopene concentration. The coefficient of determination $\left(\mathrm{R}^{2}\right)$ of the calibration curve was 0.999, with a limit of detection (LOD) and limit of quantitation (LOQ) of 0.31 and $0.94 \mu \mathrm{M}$, respectively. The LOD and LOQ were calculated based off of the standard deviation of the 


\section{LWT - Food Science and Technology}

intercept and slope, based on Validation of Analytical Procedures Methodology Q2B ICHHT (2005).

\subsection{Electron microscopy imaging}

Transmission electron microscopy (TEM) was used to assess the effect of treatment on tomato peel structure. A non-extracted ground tomato peel sample, optimally treated samples (low EA) and 30-min conventionally extracted samples were imaged by the Purdue Life Science Microscopy Facility (West Lafayette, IN). Processed tomato peels were received in acetone, transferred to fresh acetone containing $0.01 \mathrm{~g} \mathrm{~mL}^{-1}$ osmium tetroxide. After several rinses in fresh acetone they were embedded in EMbed-812 resin. Thin sections were cut on a ReichertJung Ultracut $\mathrm{E}$ ultramicrotome and stained with $0.02 \mathrm{~g} \mathrm{~mL}^{-1}$ uranyl acetate and lead citrate. Images were acquired on a FEI Tecnai T20 electron microscope equipped with a $\mathrm{LaB}_{6}$ source and operating at $200 \mathrm{kV}$.

Since the ground tomato peels used for this experiment were previously processed, fresh tomatoes were sampled from a local grocery store and used as a reference for tomato structure. These were fixed in $0.025 \mathrm{~g} \mathrm{~mL}^{-1}$ glutaraldehyde in $100 \mathrm{~mol} \mathrm{~mL}^{-1}$ sodium cacodylate buffer, post-fixed in buffered $0.01 \mathrm{~g} \mathrm{~mL}^{-1}$ osmium tetroxide containing $0.008 \mathrm{mg} \mathrm{mL}^{-1}$ potassium ferricyanide, dehydrated with a graded series of ethanol, and embedded in EMbed-812 resin. Thin sections were cut stained, and visualized following the same protocol as done for the ground tomato peels.

\subsection{Statistical analysis}

Statistical analysis was conducted with JMP version 10 (SAS Institute Inc. 2012 Cary, NC). Data was subjected to analysis of variance (ANOVA) where factors and values were considered significant at $P<0.05$. Pairwise comparisons between control and optimized extraction yields were conducted post-hoc using the Tukey-Kramer method $(\alpha=0.05)$. Lycopene content was expressed as $\mathrm{mg} / 100 \mathrm{~g}$ dry weight and each data point is represented by the mean values and standard deviation of three independent extractions.

\section{Results and discussion}

3.1. Lycopene recovery of low EA extractions

In all MAE extractions, the primary compound was all-trans-lycopene (Fig. 1). Only two parameter estimates, interaction effects solvent type $\times$ power and power $\times$ energy were significant at the $\alpha=0.05$ level, while the solid-liquid ratio did not appear to significantly $(P=0.330)$ affect the all-trans-lycopene extraction yield. Based on the RSM the maximum predicted extraction yield of all-trans-lycopene was determined to be $10.362 \mathrm{mg} / 100 \mathrm{~g}$ (Fig. 2) with a solvent ratio of 1:1 treated for $15 \mathrm{~s}(24 \mathrm{~kJ}$ equivalents) at $1600 \mathrm{~W}$. 


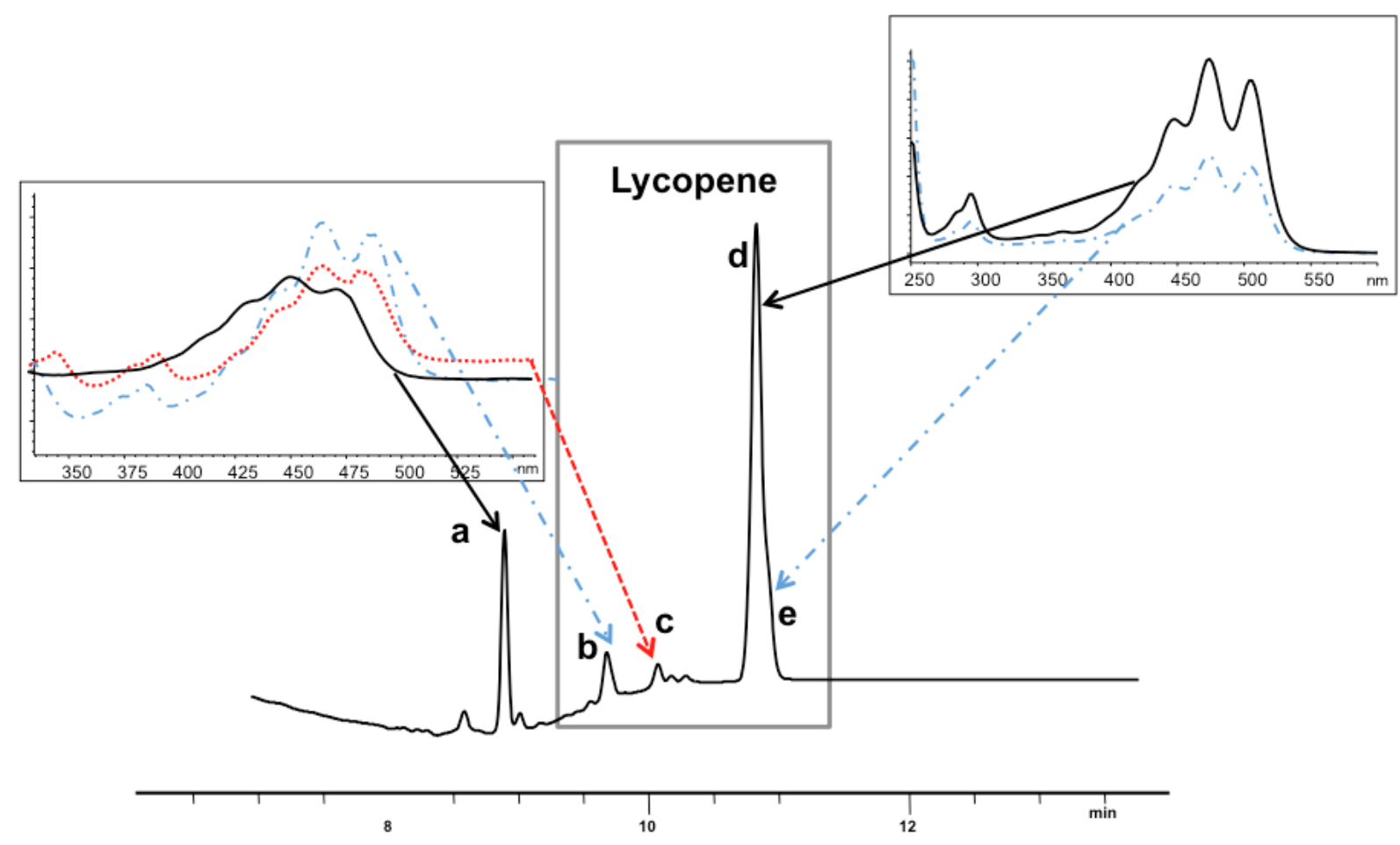

Fig. 1. Representative chromatogram of carotenoid extract from MAE of tomato peels at $470 \mathrm{~nm}$. Suspected peak identies are as follow: (a) $\beta$-carotene, (b) cis-lycopene isomer, (c) cis-lycopene isomer, (d) all-trans-lycopene, (e) 5-cis-lycopene. 

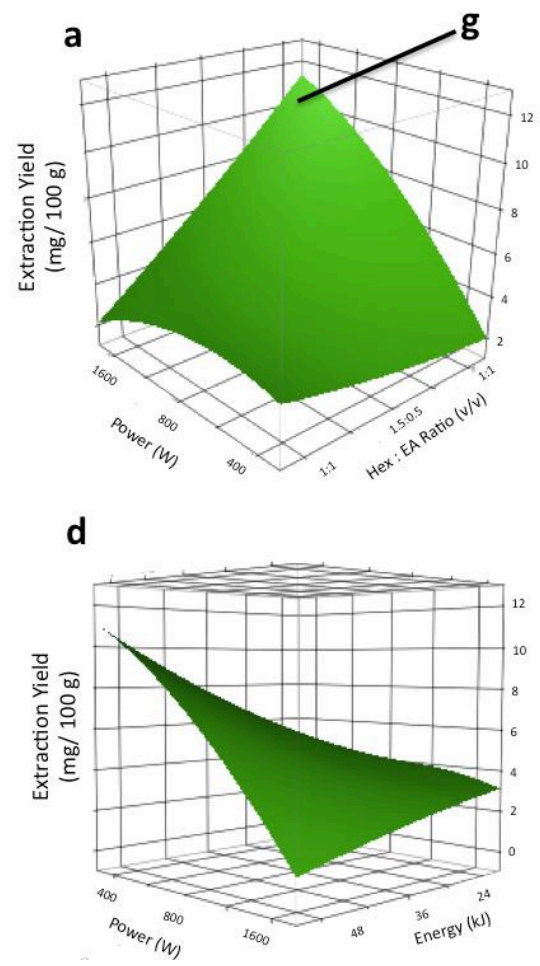

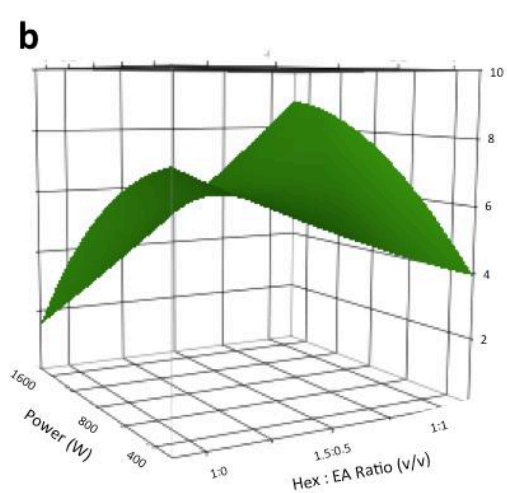

e

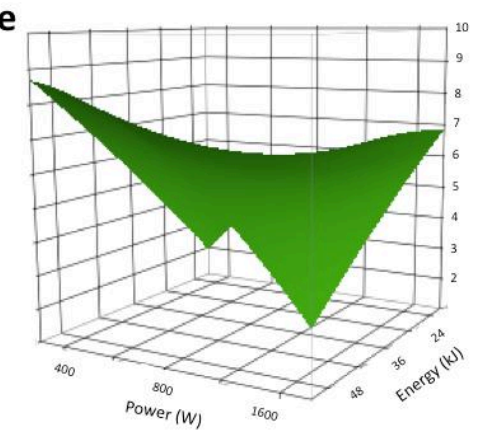

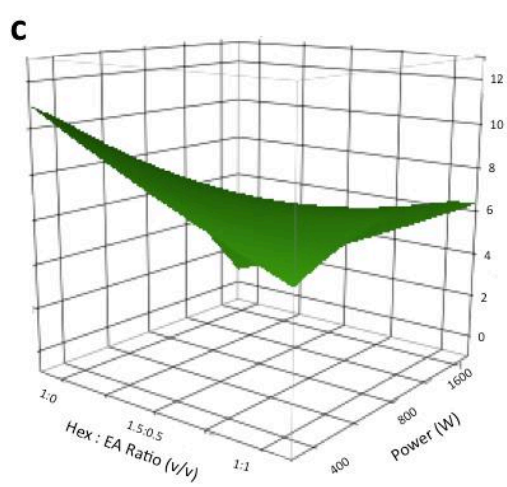

f

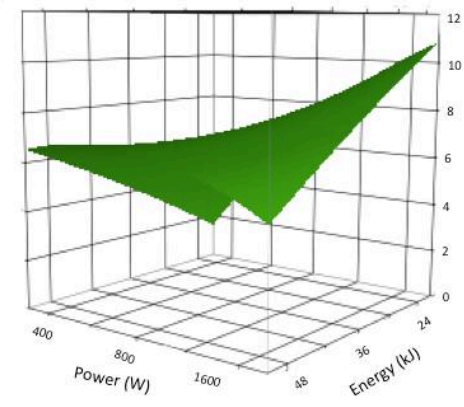

Fig. 2. Response surface plots for all-trans-lycopene yield from low EA MAE with solvent ratio vs. power (top row) and energy vs. power (bottom row) plotted. Power levels are fixed at (a) $24 \mathrm{~kJ}$, (b) $36 \mathrm{~kJ}$, and (c) $48 \mathrm{~kJ}$ and solvent ratios are fixed at (d) 1:0 hexane:EA, (e) 1.5:0.5 hexane:EA, and (f) 1:1 mL hexane:mL EA solvent ratio. The maximum predicted extraction yield was $(\mathrm{g}) 10.362 \mathrm{mg} / 100 \mathrm{~g}$ with a treatment comprising of: $1: 1 \mathrm{~mL}$ hexane:mL EA solvent ratio, $1600 \mathrm{~W}, 24 \mathrm{~kJ}$. Plotted response values represent predicted values from the model.

Statistical analysis of $c i s$-isomer extraction yield indicated that the solid-liquid ratio and the interaction effect of solvent ratio*solid-liquid ratio were significant. This suggests that cisisomer yields are increased as the solid-liquid ratio decreases and the EA proportion increases (Fig. 3). In most cases (treatments 4 vs. 22, 3 vs. 23, 5 vs. 25, and 8 vs. 24), solvent ratio with a higher proportion of EA was shown to increase the $\%$ cis yield (Table 2). No parameters were found to be significant for affecting total lycopene yield. 


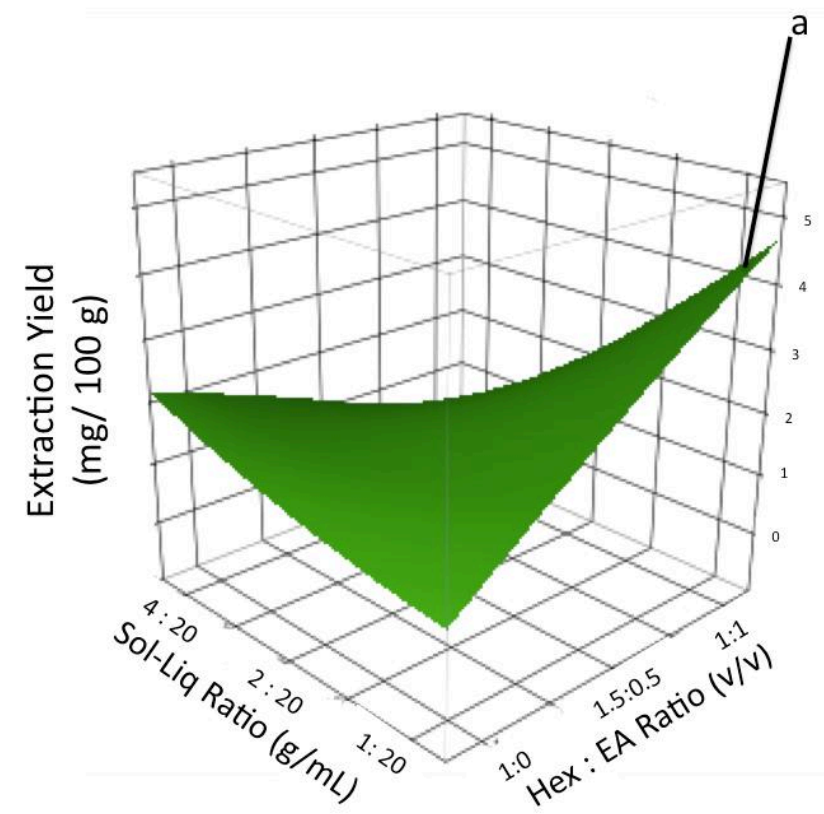

Fig. 3. Response surface plot for cis-lycopene yield from low EA MAE. The maximum cisisomer extraction yield was predicted to be (a) $4.450 \mathrm{mg} / 100 \mathrm{~g}$ with a solvent ratio of $1: 1 \mathrm{~mL}$ hexane:mL EA and a 1:20 solid-liquid ratio.

Table 2. Cis, trans, and total lycopene yields from low EA MAE

\begin{tabular}{|c|c|c|c|c|c|c|c|c|c|c|c|c|}
\hline \multirow{3}{*}{$\begin{array}{c}\begin{array}{c}\text { Treatment } \\
\text { No. }\end{array} \\
1\end{array}$} & \multirow{3}{*}{$\begin{array}{c}\begin{array}{c}\text { Coded } \\
\text { factors } \\
\left(\mathbf{X}_{\mathbf{1}}-\mathbf{X}_{\mathbf{4}}\right)\end{array} \\
0+0-\end{array}$} & \multicolumn{11}{|c|}{ Lycopene Yield mg/100 g } \\
\hline & & \multicolumn{3}{|c|}{ Cis Isomers } & \multirow{2}{*}{$\frac{\text { \% cis }}{15.339}$} & \multicolumn{3}{|c|}{ Trans } & \multirow{2}{*}{$\begin{array}{c}\text { \% trans } \\
82.943\end{array}$} & \multicolumn{3}{|c|}{ Total } \\
\hline & & 1.429 & \pm & 0.04 & & 7.727 & \pm & 0.251 & & 9.316 & \pm & 0.289 \\
\hline 2 & 0000 & 1.701 & \pm & 0.555 & 21.247 & 6.615 & \pm & 1.207 & 82.626 & 8.006 & \pm & 1.193 \\
\hline 3 & $-00+$ & 2.128 & \pm & 0.102 & 19.460 & 8.499 & \pm & 1.033 & 77.723 & 10.935 & \pm & 0.994 \\
\hline 4 & --00 & 0.537 & \pm & 0.363 & 8.894 & 5.501 & \pm & 1.08 & 91.106 & 6.038 & \pm & 1.399 \\
\hline 5 & $-0-0$ & 1.791 & \pm & 0.39 & 17.949 & 7.877 & \pm & 1.7 & 78.944 & 9.978 & \pm & 2.094 \\
\hline 6 & 0000 & 1.838 & \pm & 0.428 & 20.696 & 6.73 & \pm & 0.668 & 75.780 & 8.881 & \pm & 1.088 \\
\hline 7 & $00+-$ & 1.675 & \pm & 0.328 & 20.269 & 6.278 & \pm & 0.533 & 75.968 & 8.264 & \pm & 0.359 \\
\hline 8 & $-00-$ & 1.878 & \pm & 0.223 & 22.128 & 6.295 & \pm & 0.568 & 74.172 & 8.487 & \pm & 0.774 \\
\hline 9 & $0++0$ & 1.27 & \pm & 0.026 & 16.920 & 6.079 & \pm & 1.451 & 80.989 & 7.506 & \pm & 1.479 \\
\hline 10 & 0000 & 2.039 & \pm & 0.05 & 23.442 & 6.339 & \pm & 1.474 & 72.879 & 8.698 & \pm & 1.445 \\
\hline 11 & $+0+0$ & 2.38 & \pm & 0.031 & 19.883 & 9.279 & \pm & 0.864 & 77.519 & 11.97 & \pm & 0.898 \\
\hline 12 & $0-0-$ & 3.012 & \pm & 1.652 & 27.253 & 7.397 & \pm & 0.67 & 66.929 & 11.052 & \pm & 2.279 \\
\hline 13 & $00-+$ & 2.065 & \pm & 0.197 & 21.907 & 7.041 & \pm & 0.743 & 74.698 & 9.426 & \pm & 0.632 \\
\hline 14 & $0+-0$ & 1.178 & \pm & 0.177 & 18.134 & 5.16 & \pm & 0.468 & 79.433 & 6.496 & \pm & 0.537 \\
\hline 15 & $0-0+$ & 3.946 & \pm & 0.245 & 34.111 & 6.999 & \pm & 1.45 & 60.503 & 11.568 & \pm & 1.608 \\
\hline 16 & $0-+0$ & 1.676 & \pm & 1.747 & 22.881 & 5.043 & \pm & 0.546 & 68.846 & 7.325 & \pm & 2.211 \\
\hline 17 & $0+0+$ & 1.103 & \pm & 0.19 & 19.546 & 4.383 & \pm & 0.431 & 77.671 & 5.643 & \pm & 0.459 \\
\hline 18 & $0--0$ & 2.209 & \pm & 1.38 & 30.269 & 4.473 & \pm & 0.914 & 61.291 & 7.298 & \pm & 2.289 \\
\hline 19 & $-0+0$ & 1.295 & \pm & 0.672 & 24.160 & 3.752 & \pm & 0.693 & 70.000 & 5.36 & \pm & 1.357 \\
\hline 20 & -+00 & 1.192 & \pm & 0.037 & 21.214 & 4.268 & \pm & 0.198 & 75.957 & 5.619 & \pm & 0.222 \\
\hline 21 & $00++$ & 0.873 & \pm & 0.069 & 27.758 & 2.802 & \pm & 0.525 & 89.094 & 3.145 & \pm & 0.631 \\
\hline 22 & +-00 & 4.174 & \pm & 0.495 & 34.160 & 7.435 & \pm & 0.276 & 60.848 & 12.219 & \pm & 0.266 \\
\hline 23 & $+00+$ & 1.721 & \pm & 0.152 & 23.057 & 5.43 & \pm & 1.031 & 72.749 & 7.464 & \pm & 1.131 \\
\hline 24 & +00 & 1.546 & \pm & 0.097 & 19.513 & 6.064 & \pm & 1.509 & 76.537 & 7.923 & \pm & 1.608 \\
\hline 25 & $+0-0$ & 1.677 & \pm & 0.506 & 20.137 & 6.344 & \pm & 0.84 & 76.177 & 8.328 & \pm & 1.155 \\
\hline 26 & ++00 & 1.009 & \pm & 0.069 & 20.100 & 3.855 & \pm & 0.093 & 76.793 & 5.02 & \pm & 0.157 \\
\hline 27 & 00-- & 1.391 & \pm & 0.454 & 26.225 & 3.594 & \pm & 0.097 & 67.760 & 5.304 & \pm & 0.451 \\
\hline
\end{tabular}


Comparison between lycopene yields of the predicted optimized MAE treatment (1:1 solvent ratio, 1:0 solid-liquid ratio, $1600 \mathrm{~W}, 24 \mathrm{~kJ}$ equivalents done for $15 \mathrm{~s}$ ) vs. conventional treatment conducted at the same time and temperature in a shaking water bath indicated that MAE exhibited a significantly greater all-trans-lycopene yield compared to the 15 -s but not the 30min conventional extraction (Fig. 4). However, no differences were found between cis-isomer and total lycopene yield.

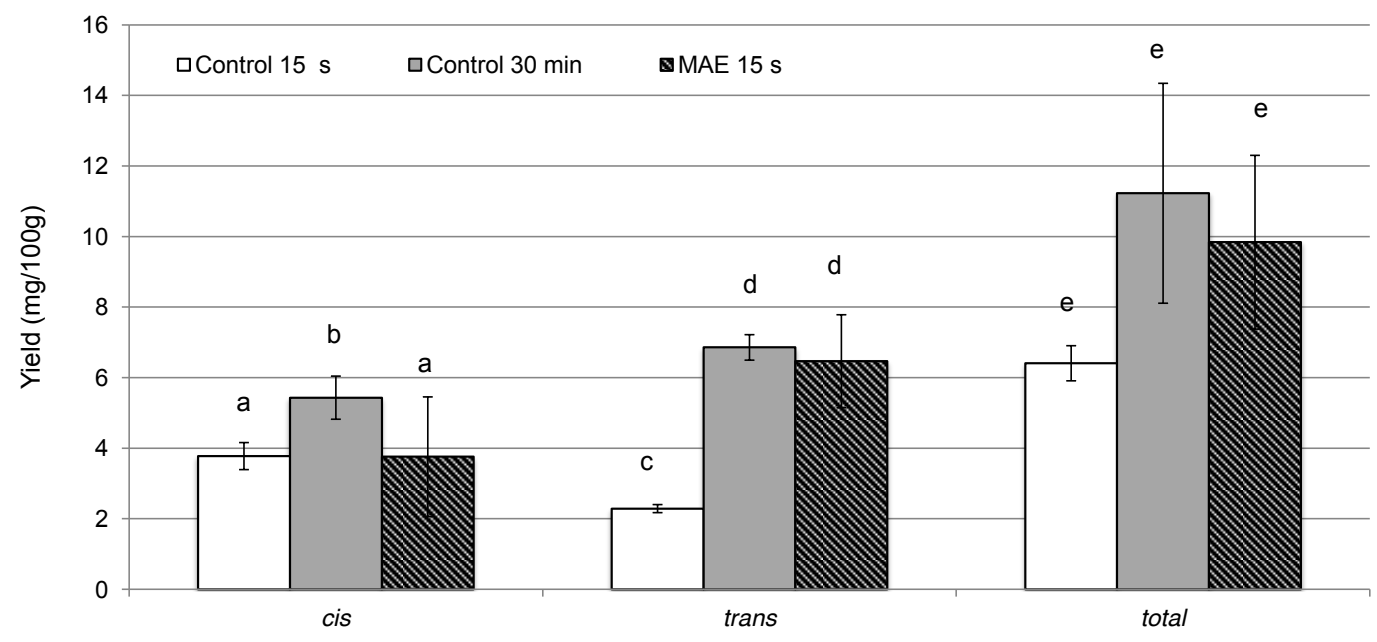

Fig. 4. Comparison of control (conventional) methods vs. optimized low EA MAE. The MAE conditions used (1:1 solvent ratio, 1:20 solid-liquid ratio, $1600 \mathrm{~W}, 24 \mathrm{~kJ}$ equivalents for $15 \mathrm{~s}$ ) were determined as optimal by RSM. Extraction yields of cis, trans, and total lycopene are shown where same letters denote values that are not significantly different at the $\alpha=0.05$ level based on the Tukey Kramer method for pairwise comparisons. Response values shown represent the mean $\pm \mathrm{SD}(n=3)$.

It should be pointed out that when the predicted optimal conditions were actually tested, the alltrans-lycopene yield obtained (Fig. 4) was less than the value predicted by the model (Fig. 2). Statistical analysis indicated that the model for all-trans-lycopene had a significant lack of fit with $P$-value $=0.0143$ and a low coefficient of determination $\left(\mathrm{R}^{2}=0.58\right)$. Treatment $11(1: 1$ solvent ratio, 2:20 solid-liquid ratio, $1600 \mathrm{~W}, 36 \mathrm{~kJ}$ equivalents with a $30 \mathrm{~s}$ treatment) had the greatest all-trans-lycopene yield at $9.279 \pm 0.864 \mathrm{mg} / 100 \mathrm{~g}$ (Table 2). This may be due to the need for additional energy, more than $24 \mathrm{~kJ}$, to extract lycopene at the given solvent and power level. A statistical drawback of the Box-Behnken design is that over interpretation due to extrapolation towards the corners of the response surface can occur. Thus, a second RSM experiment (high EA) using a CCD was conducted focusing on only two factors.

\subsection{Lycopene recovery of high EA extractions}

Since RSM demonstrated increasing all-trans-lycopene yields with increasing EA concentrations (Fig. 2), this second set of experiments employed solvent mixtures with lower hexane-to-EA ratios $(2: 8,1: 9,0: 1)$ and fixed all treatments at $24 \mathrm{~kJ}$ equivalents with $400 \mathrm{~W}(1 \mathrm{~min}), 800 \mathrm{~W}$ 
(30 s), or $1600 \mathrm{~W}(15 \mathrm{~s})$. Solid-liquid ratio (1:20) was fixed because a limited supply of sample was available. Surface plots also indicated that adjustments in power and energy could improve yields, however, the Mars Xpress microwave extraction system has only three power settings, 400,800 , and $1600 \mathrm{~W}$, thus preventing the ability to increase or decrease power. Energy was also limited as preliminary testing demonstrated that high-energy inputs caused solvent evaporation, which would effectively shut down the system for safety reasons. Since a higher proportion of EA increases the polarity of the solvent and the rate of heating, a fixed low energy equivalent $(24 \mathrm{~kJ})$ was chosen for the high EA experiments.

ANOVA of high EA MAE indicated that there was a significant difference amongst treatments $(P=0.0164)$ for all-trans-lycopene extraction. In this case, only solvent ratio was found to be a significant factor influencing the extraction yield of all-trans-lycopene. The model did not exhibit a significant lack of fit $(P=0.1624)$, and predicted a maximum yield of $13.872 \mathrm{mg} / 100 \mathrm{~g}$ (Fig. 5) for an extraction with ethyl acetate at a power of $400 \mathrm{~W}$, which for a $24 \mathrm{~kJ}$ equivalent had a treatment time of $1 \mathrm{~min}$. For cis-isomer extraction, no significant difference was found amongst treatments. However, the \% cis of the extracts was greatest for treatments 1 and 9 , which were the only ones using a solvent ratio of 2:8 (Table 3).

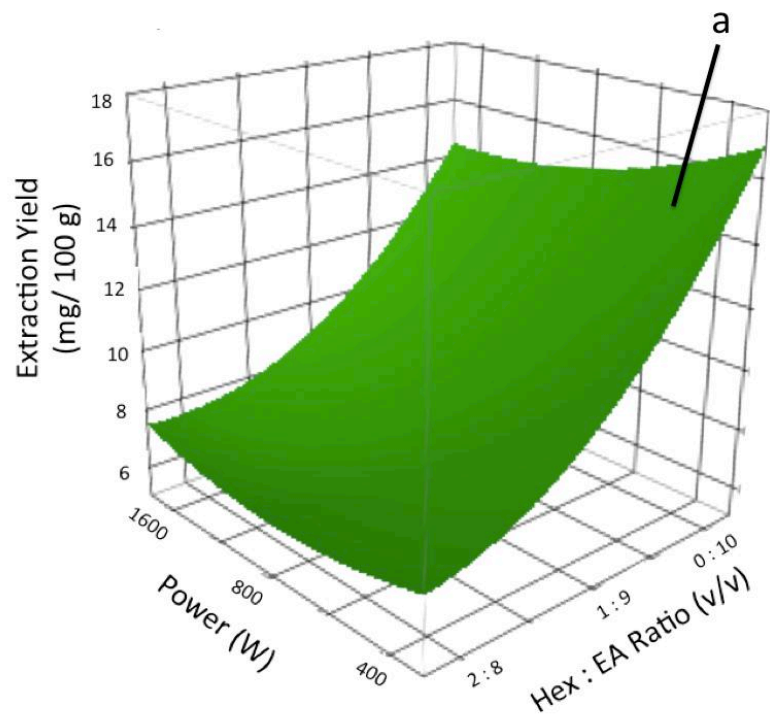

Fig. 5. Response surface plot for all-trans-lycopene yield from high EA MAE. The maximum all-trans-extraction yield was predicted to be (a) $13.872 \mathrm{mg} / 100 \mathrm{~g}$ with a full EA solvent and when treated at $400 \mathrm{~W}$. Solvent ratio significantly affected the extraction yield $(P=0.004)$ while power did not $(P=0.210)$. Plotted response values indicate mean $\pm \mathrm{SD}(\mathrm{n}=3)$. 
LWT - Food Science and Technology

Volume 62, Issue 1, Part 1, 2015, Pages 160-168

Table 3. Cis, trans, and total lycopene yields from high EA MAE.

\begin{tabular}{|c|c|c|c|c|c|c|c|c|c|c|c|c|}
\hline \multirow{3}{*}{$\begin{array}{c}\begin{array}{c}\text { Treatment } \\
\text { No. }\end{array} \\
1\end{array}$} & \multirow{3}{*}{$\begin{array}{c}\begin{array}{c}\text { Coded factors } \\
\left(\mathbf{X}_{1}-\mathbf{X}_{4}\right)\end{array} \\
--\end{array}$} & \multicolumn{11}{|c|}{ Lycopene Yield mg/100 g } \\
\hline & & \multicolumn{3}{|c|}{ Cis Isomers } & \multirow{2}{*}{$\begin{array}{c}\text { \% cis } \\
43.991\end{array}$} & \multicolumn{3}{|c|}{ Trans } & \multirow{2}{*}{$\begin{array}{c}\text { \% trans } \\
52.138\end{array}$} & \multicolumn{3}{|c|}{ Total } \\
\hline & & 3.909 & \pm & 0.243 & & 4.633 & \pm & 1.944 & & 8.886 & \pm & 1.817 \\
\hline 2 & 00 & 3.857 & \pm & 2.336 & 39.490 & 5.562 & \pm & 2.956 & 56.947 & 9.767 & \pm & 0.621 \\
\hline 3 & +- & 3.44 & \pm & 0.636 & 19.799 & 12.195 & \pm & 0.884 & 70.187 & 17.375 & \pm & 0.253 \\
\hline 4 & -0 & 3.409 & \pm & 0.436 & 35.378 & 5.891 & \pm & 0.41 & 61.135 & 9.636 & \pm & 0.851 \\
\hline 5 & $0-$ & 3.288 & \pm & 0.898 & 25.980 & 9.028 & \pm & 0.178 & 71.334 & 12.656 & \pm & 0.867 \\
\hline 6 & +0 & 2.624 & \pm & 0.295 & 17.344 & 12.175 & $\overline{ \pm}$ & 1.611 & 80.475 & 15.129 & \pm & 1.892 \\
\hline 7 & $0+$ & 2.263 & \pm & 0.366 & 22.493 & 7.454 & \pm & 0.346 & 74.088 & 10.061 & \pm & 0.379 \\
\hline 8 & 00 & 2.824 & \pm & 0.998 & 30.799 & 6.002 & \pm & 0.857 & 65.460 & 9.169 & \pm & 0.205 \\
\hline 9 & -+ & 3.753 & \pm & 0.082 & 42.287 & 4.788 & \pm & 0.461 & 53.949 & 8.875 & \pm & 0.42 \\
\hline 10 & ++ & 3.577 & \pm & 0.806 & 24.470 & 10.711 & \pm & 0.671 & 73.273 & 14.618 & \pm & 1.471 \\
\hline
\end{tabular}

The actual optimal all-trans-lycopene yield was determined as $13.592 \mathrm{mg} / 100 \mathrm{~g}$ (Fig. 6), which was statistically greater than the $1 \mathrm{~min}(P=0.0006)$ and 30 -min conventional extraction $(P<0.0001)$. Similarly, the total lycopene yield was significantly greater for the optimized MAE treatment compared to the 30 -min control $(P<0.0001)$ and conventional control $(P=0.006)$. Significant differences were not observed amongst treatments for cis-isomer yield $(P>0.05)$. However, the proportion of cis-isomers to extracted trans-lycopene is dramatically higher for the 30-min control compared to the 1-min control and the optimized MAE treatment. The relative increase in cis-isomers may be due to the longer treatment time.

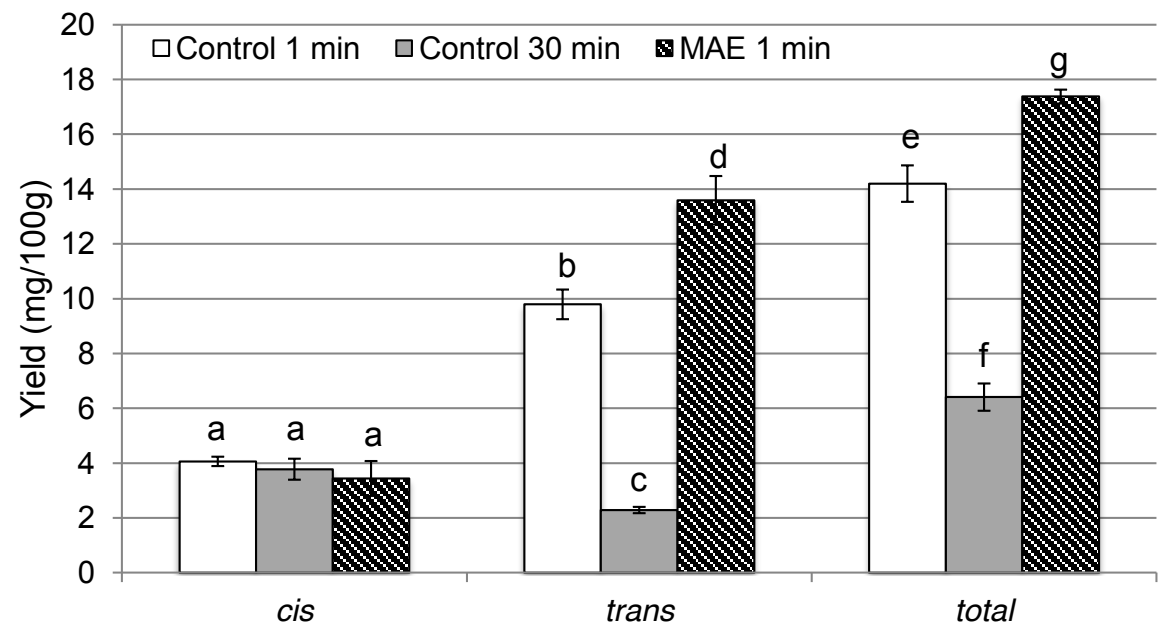

Fig. 6. Comparison of control (conventional) methods vs. the high EA MAE treatment with the highest all-trans-lycopene yield. The MAE conditions used (0:1 solvent ratio, 1:20 solid-liquid ratio, $400 \mathrm{~W}, 24 \mathrm{~kJ}$ equivalents for $60 \mathrm{~s}$ ) were determined as optimal by RSM. Extraction yields

of cis, trans, and total lycopene are shown where same letters denote values that are not significantly different at the $\alpha=0.05$ level based on the Tukey Kramer method for pairwise comparisons. Response values shown represent the mean $\pm \operatorname{SD}(n=3)$. 
The literature reports different lycopene recoveries depending on the extraction method and type of raw material. Enzyme assisted extraction, was found to be extremely efficient with $440 \mathrm{mg} / 100 \mathrm{~g}$ of lycopene from tomatoes (Lavecchia \& Zuorro, 2008), although the process can be costly. It should also be noted that whole tomatoes may contain more lycopene since they have not been previously processed. Studies done on tomato peels reported yields ranging from 0.639 to $73.40 \mathrm{mg} / 100 \mathrm{~g}$ (Kaur et al., 2008, Knoblich et al., 2005, Shi et al., 2009). Kaur et al. (2008) found that a maximum recovery of $1.98 \mathrm{mg}$ lycopene $/ 100 \mathrm{~g}$ was attainable when tomato skins $(0.15 \mathrm{~mm}$ particle size $)$ were conventionally extracted with hexane:acetone:alcohol (2:1:1 mL:mL:mL) with $0.5 \mathrm{mg} \mathrm{mL}^{-1}$ BHT at a $1: 30$ solid-liquid ratio $(\mathrm{w} / \mathrm{v})$, at $50{ }^{\circ} \mathrm{C}$ for $8 \mathrm{~min}$ four times. Specifically, lycopene yield increased as a function of extraction number (repeated on one sample) and decreasing particle size. The authors hypothesized that the extractions conducted at $50^{\circ} \mathrm{C}$ allowed for better breakdown of chromoplast membranes compared to cooler conditions, yet did not induce significant degradation compared to treatments done at $60{ }^{\circ} \mathrm{C}$. Shi et al. (2009) determined a higher total lycopene content in dried tomato skins at $\sim 13.0 \mathrm{mg} / 100 \mathrm{~g}$ when extracted with hexane overnight at $45^{\circ} \mathrm{C}$. The authors compared this to supercritical fluid extraction and achieved a maximum recovery of $73.3 \%$ when ethanol and olive oil were used as modifiers at $75^{\circ} \mathrm{C}$ and $35 \mathrm{MPa}$, which is less efficient compared to the results in this study. Although the results presented in this study from MAE are likely an improvement over conventional solvent extraction and over supercritical fluid extraction, lycopene yields were still significantly lower than expected. Lower yields in this study may be partially due to previous processing (i.e. hot break) that peels underwent, which lowered the amount of extractable lycopene (Kaur et al., 2008) or due to differences in tomato variety (George et al., 2004).

Tomato peels following treatments (MAE and conventional) were still visibly orange, suggesting that lycopene remained in the peel as a non-extractable fraction. Calvo, Dado, and Santa-Maria (2007) encountered a similar result when they heated freeze-dried tomato peels in ethanol or ethyl acetate at temperatures ranging from 25 to $60^{\circ} \mathrm{C}$. Ethanol was found to have a greater lycopene extraction yield, possibly due to its ability to better penetrate the peels compared to ethyl acetate, however, residual pigment remained in the sample following treatment. Attempts were made to remove all the apparent color from the tomato peels (data not shown), however none of the procedures were able to completely remove pigments. A well established extraction procedure involving sonication of chloroform-soaked peels (Jun, 2006, Naviglio et al., 2008, Rozzi et al., 2002) was tested, however, the treated peels exhibited little noticeable color following treatment and yielded low lycopene concentrations. Modifications were also made to the extraction procedure following methods done previously by Goltz et al. (2012) by testing different solvent types and ratios, further reducing particle size with a high shear mixer, and using caustic pretreatments (done at various times and temperatures) with $0.4 \mathrm{~g} \mathrm{~mL}^{-1}$ potassium hydroxide in methanol. However, the latter resulted in the pigment loss into the aqueous phase, due to either degradation of the carotenoids (exhibited by lack of color or the development of a dark color). To determine absolute lycopene content in tomato peels, future studies require treatments that can effectively disrupt or degrade the physical cell structure barriers without affecting embedded lycopene. 


\subsection{Transmission electron microscopy of tomato peels}

TEM micrographs were unable to display cell ultrastructure of byproduct tomato peels, possibly due to the extent of processing the peels underwent prior to receiving. However, the ultrastructure with lycopene bodies can be seen in the fresh tomato peel samples as the spherical electron dense (dark) regions (Fig. 7a). The non-treated byproduct tomato peel (Fig. 7b) appeared to be significantly less damaged compared to treated peels (Fig. 7c and d). In particular, the MAE (1:1 solvent ratio, 1:20 solid-liquid ratio, $1600 \mathrm{~W}, 24 \mathrm{~kJ}$, for $15 \mathrm{~s}$ ) treated samples exhibited significantly more structural disruption as fissures and gray wholes appeared to be more prevalent. This suggests that the MAE was better able to disrupt cellular structure to reduce physical extraction barriers.
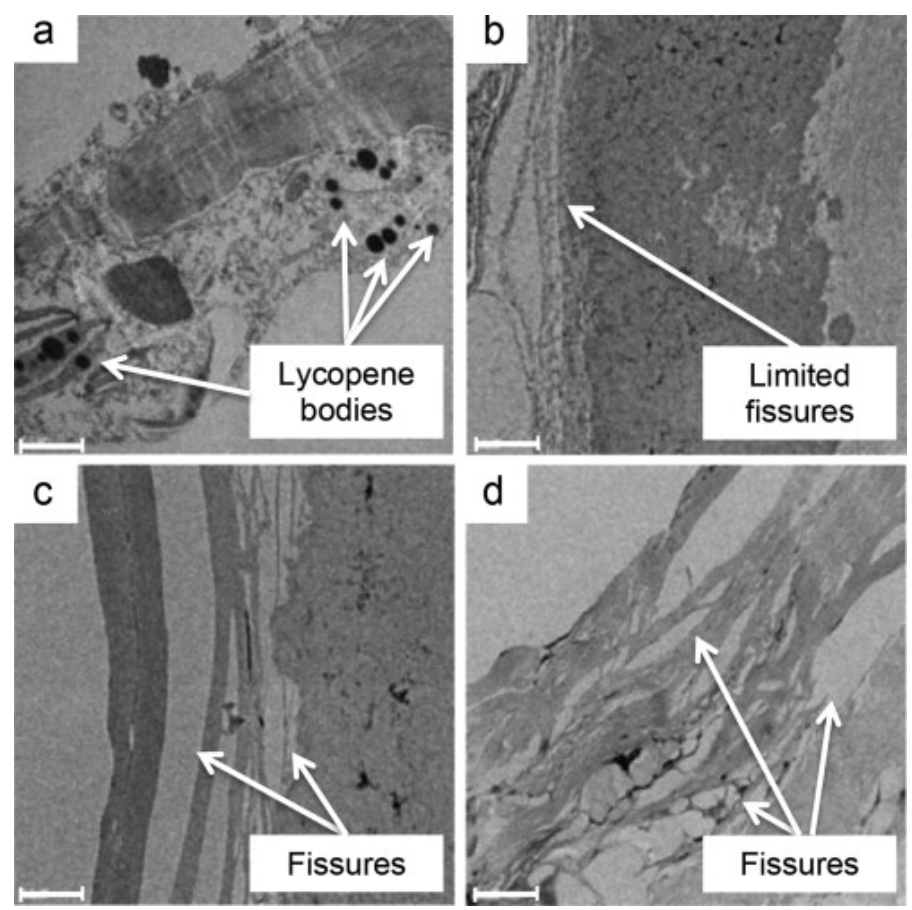

Fig. 7. TEM images of tomato peels following (a) fresh tomato peel with no extraction, (b) byproduct tomato peel with no extraction, c) control extraction for $30 \mathrm{~min}$, and (d) MAE (1:1 solvent ratio, 1:20 solid-liquid ratio, $1600 \mathrm{~W}, 24 \mathrm{~kJ}$, for $15 \mathrm{~s}$ ). Visibly more holes and fissures are present in extracted samples, thus suggesting that MAE, and to some extent conventional extraction, cause structural disruption. Scale bars indicate $1 \mu \mathrm{m}$.

\section{Conclusions}

Optimization data indicated that solvent ratio and microwave power in relation to energy equivalents significantly affected the all-trans-lycopene extraction yield. Cis-isomer extraction was primarily affected by the solvent ratio and solid-liquid ratio. The maximum all-translycopene yield of $\sim 13 \mathrm{mg} / 100 \mathrm{~g}$ was obtained with ethyl acetate at $400 \mathrm{~W}$, with a $24 \mathrm{~kJ}$ equivalent $(1 \mathrm{~min})$. Significantly more all-trans-lycopene was extracted with ethyl acetate via MAE compared to a 1-min and 30-min conventional treatment. TEM suggested that selective, 
physical disruption occurs in the tomato peels during MAE. All-trans-lycopene has been of interest for food and pharmaceutical industries since it is the most stable isomer. Additionally, all-trans-lycopene exhibits greater color intensity compared to cis-isomers due to a hypsochromic shift and smaller extinction coefficient of the latter (Schieber \& Carle, 2005). However, interest in cis-lycopene is growing as there is some evidence indicating that these isomers are more bioavailable compared to the all-trans form (Boileau, Boileau, \& Erdman, 2002). Although certain limitations to MAE exist (i.e. consumer preference against solvent use and challenges with scaling up) the findings of this study offer applicable information that could steer other extraction techniques towards cis or trans-isomer recovery, depending on the application.

\section{Acknowledgments}

This material is based upon work supported by the National Science Foundation Graduate Research Fellowship under Grant No. DGE-1333468 and the Purdue Department of Food Science Industry Fellows Program. The authors thank Red Gold (IN, USA) for providing tomato peels, Mohneet Ahuja from the Purdue University Statistical Consulting Service for advice on statistical design and Laurie Mueller from the Purdue Life Science Microscopy Facility for her expertise with TEM.

\section{References}

S. Agarwal, A.V. Rao. Tomato lycopene and its role in human health and chronic disease Canadian Medical Association, 163 (6) (2000), pp. 739-744

H. Al-Wandawi, M. Abdul-Rahman, K. Al-ShaikhlyTomato processing wastes as essential raw materials source Journal of Agricultural and Food Chemistry, 33 (5) (1985), pp. 804-807, 10.1021/jf00065a009

A. Ascenso, S. Pinho, C. Eleutério, F.G. Praça, M.V.L.B. Bentley, H. Oliveira, et al. Lycopene from tomatoes: vesicular nanocarrier formulations for dermal delivery Journal of Agricultural and Food Chemistry, 61 (30) (2013), pp. 7284-7293, 10.1021/jf401368w

A. Baiano, L. Bevilacqua, C. Terracone, F. Contò, M.A. Del Nobile Single and interactive effects of process variables on microwave-assisted and conventional extractions of antioxidants from vegetable solid wastes Journal of Food Engineering, 120 (0) (2014), pp. 135-145 http://dx.doi.org/10.1016/j.jfoodeng.2013.07.010

T.W.-M. Boileau, A.C. Boileau, J.W. Erdman Bioavailability of all-trans and cis-isomers of lycopene Experimental Biology and Medicine, 227 (10) (2002), pp. 914-919

M.M. Calvo, D. Dado, G. Santa-Maria Influence of extraction with ethanol or ethyl acetate on the yield of lycopene, $\beta$-carotene, phytoene, and phytofluene from tomato peel powder European Food Research Technology, 224 (1) (2007), pp. 567-571, $10.1007 / \mathrm{s} 00217-006-0335-8$ 
J. Chen, J. Shi, S.J. Xue, Y. Ma Comparison of lycopene stability in water- and oil-based food model systems under thermal- and light-irradiation treatmentsLWT - Food Science and Technology, 42 (3) (2009), pp. 740-747 http://dx.doi.org/10.1016/j.lwt.2008.10.002

D.V. Dandekar, V.G. Gaikar Microwave assisted extraction of curcuminoids from Curcuma longa Separation Science and Technology, 37 (11) (2002), pp. 2669-2690, 10.1081/SS$\underline{120004458}$

P. Di Mascio, S. Kaiser, H. Sies Lycopene as the most efficient biological carotenoid singlet oxygen quencher Archives of Biochemistry and Biophysics, 274 (2) (1989), pp. 532-538 http://dx.doi.org/10.1016/0003-9861(89)90467-0

Economic Research Service, United States Department Agriculture U.S. Tomato Statistics (92010)(2010) Online: Retrieved from http://usda.mannlib.cornell.edu/MannUsda/viewDocumentInfo.do?documentID=1210

B. George, C. Kaur, D.S. Khurdiya, H.C. KapoorAntioxidants in tomato (Lycopersium esculentum) as a function of genotype Food Chemistry, 84 (1) (2004), pp. 45-51 http://dx.doi.org/10.1016/S0308-8146(03)00165-1

S.R. Goltz, W.W. Campbell, C. Chitchumroonchokchai, M.L. Failla, M.G. Ferruzzi Meal triacylglycerol profile modulates postprandial absorption of carotenoids in humans Molecular Nutrition \& Food Research, 56 (2012), pp. 866-877, 10.1002/mnfr.201100687

W.M. Harris, A.R. SpurrChromoplasts of tomato fruits. II. The red tomato American Journal of Botany, 56 (4) (1969), pp. 380-389, 10.2307/2440813

ICHHT, Guideline Validation of analytical procedures: Text and methodology Q2 (R1) IFPMA, Geneva (2005)

X. Jun Application of high hydrostatic pressure processing of food to extracting lycopene from tomato paste waste High Pressure Research, 26 (1) (2006), pp. 33-41, $10.1080 / 08957950600608741$

D. Kaur, A.A. Wani, D.P.S. Oberoi, D.S. Sogi Effect of extraction conditions on lycopene extractions from tomato processing waste skin using response surface methodology Food Chemistry, 108 (2) (2008), pp. 711-718 http://dx.doi.org/10.1016/j.foodchem.2007.11.002

E.G. Kean, B.R. Hamaker, M.G. Ferruzzi Carotenoid bioaccessibility from whole grain and degermed maize meal products Journal of Agricultural and Food Chemistry, 56 (21) (2008), pp. 9918-9926, 10.1021/jf8018613

M. Knoblich, B. Anderson, D. Latshaw Analyses of tomato peel and seed byproducts and 
their use as a source of carotenoids Journal of the Science of Food and Agriculture, 85 (7) (2005), pp. 1166-1170

R. Lavecchia, A. Zuorro Improved lycopene extraction from tomato peels using cell-wall degrading enzymes European Food Research and Technology, 228 (1) (2008), pp. 153$158,10.1007 / \mathrm{s} 00217-008-0897-8$

D. Naviglio, T. Caruso, P. Iannece, A. Aragòn, A. Santini Characterization of high purity lycopene from tomato wastes using a new pressurized extraction approach Journal of Agricultural and Food Chemistry, 56 (15) (2008), pp. 6227-6231, 10.1021/jf703788c

E.D. Neas, M.J. Collins Introduction to microwave sample preparation H.M. Kingston, L.B. Jassie (Eds.), American Chemical Society, Washington, D.C. (1988), pp. 7-32

M.L. Nguyen, S.J. Schwartz Lycopene stability during food processing Experimental Biology and Medicine, 218 (2) (1998), pp. 101-105

D.B. Rodriguez-Amaya A guide to carotenoid analysis in foods ILSI Press, Washington, DC (2001)

N.L. Rozzi, R.K. Singh, R.A. Vierling, B.A. Watkins Supercritical fluid extraction of lycopene

from tomato processing byproducts Journal of Agricultural and Food Chemistry, 50 (9) (2002), pp. 2638-2643, 10.1021/jf011001t

A. Schieber, R. Carle Occurrence of carotenoid cis-isomers in food: technological, analytical, and nutritional implications Trends in Food Science \& Technology, 16 (9) (2005), pp. 416-422 http://dx.doi.org/10.1016/j.tifs.2005.03.018

J. Shi, Y. Dai, Y. Kakuda, G. Mittal, S.J. Xue Effect of heating and exposure to light on the stability of lycopene in tomato puree Food Control, 19 (5) (2008), pp. 514-520

J. Shi, C. Yi, S.J. Xue, Y. Jiang, Y. Ma, D. Li Effects of modifiers on the profile of lycopene extracted from tomato skins by supercritical CO2 Journal of Food Engineering, 93 (4) (2009), pp. 431-436 http://dx.doi.org/10.1016/j.jfoodeng.2009.02.008

I.F. Strati, V. Oreopoulou Process optimisation for recovery of carotenoids from tomato waste Food Chemistry, 129 (3) (2011), pp. 747-752 http://dx.doi.org/10.1016/j.foodchem.2011.05.015

S. Thornsbury Tomatoes USDA Economic Research Service (2012)

T. Zou, D. Wang, H. Guo, Y. Zhu, X. Luo, F. Liu, et al. Optimization of microwave-assisted extraction of anthocyanins from mulberry and identification of anthocyanins in extract using HPLC-ESI-MS Journal of Food Science, 77 (1) (2012), pp. C46-c50, 10.1111/j.1750-3841.2011.02447.x 